\title{
Administración eficiente de impuestos para el acceso a las cadenas globales de suministro del sector automotriz en Colombia: una visión contable
}

\author{
Kevin Hermann, Fernando Salazar-Arrieta*, Héctor A. Garzón y Braulio A. Rodríguez \\ Pontificia Universidad Javeriana, Facultad de Ciencias Económicas y Administrativas, Bogotá, D.C.-Colombia. \\ (correo-e: khermann@javeriana.edu.co, salazar.fernando@javeriana.edu.co, h.garzon@javeriana.edu.co, \\ brodri@javeriana.edu.co).
}

Recibido May. 11, 2020; Aceptado Jul. 9, 2020; Versión final Sep. 8, 2020, Publicado Dic. 2020

\begin{abstract}
Resumen
Este estudio tiene como objetivo delimitar los efectos y las aplicaciones de la administración eficiente de los impuestos, como soporte para el acceso a las cadenas globales de suministro (CGS) por parte de la industria automotriz colombiana. Metodológicamente, se analizan las principales características del sector en otros países de la región y su inserción en estas cadenas globales desde la administración eficiente de impuestos y la gestión financiera de las compañías. Se evidencian ventajas competitivas y eficiencias fiscales, que al ser incorporadas en la legislación adoptan un análisis para cada jurisdicción. Los resultados muestran la necesidad de análisis financiero, que permita la incorporación de competencias para manejar información transversal en las dimensiones de competitividad, fortalecimiento y valor agregado. Se concluye que tener en cuenta la evolución del concepto de cadenas de suministros (CS) y CGS permite observar de forma general la función que cumplen las CS en la generación de ventajas competitivas para las compañías.
\end{abstract}

Palabras clave: cadenas globales de suministro; competitividad; administración fiscal; jurisdicción; valor agregado

\section{Efficient tax administration for access to global supply chains by the automotive sector in Colombia: an accounting vision}

\begin{abstract}
The objective of this study is to define the effects and applications of efficient tax administration as support for access to global supply chains (GSC) by the Colombian automotive industry. Methodologically, the main characteristics of the automotive sector in other countries of the region and their insertions in global chains are examined from the efficient administration of taxes and the financial management of companies. There are competitive advantages and fiscal efficiencies, which when incorporated into the legislation provide an analysis for each jurisdiction. The results show the need for financial analysis, which allows the incorporation of competencies to handle cross-sectional information in the dimensions of competitiveness, strengthening, and added value. It is concluded that considering the concept evolution of supply chains (SC) and GSCs allows observing, in a general manner, the role that CSs have in the generation of competitive advantages for companies.
\end{abstract}




\section{INTRODUCCIÓN}

El proyecto de investigación que da origen al presente artículo, parte de un enfoque cualitativo, en el que se pretende comprender los ejes que orientan el objeto de estudio en la administración eficiente de impuestos de la industria automotriz en Colombia, cuyo alcance es descriptivo en cuanto se definieron los conceptos y se analizó la relación del objeto de estudio con el ingreso a las cadenas globales de suministro, para describir en este contexto la inclusión a las Cadenas Globales de Suministro y cómo desde la profesión contable es necesario apoyar los procesos tributarios y de fiscalidad y sus problemáticas. La Organización Mundial de Comercio (OMC, 1994) junto con otros organismos internacionales como la Organización para la Cooperación y el Desarrollo Económico (OCDE, 2014) y el Grupo del Banco Mundial (WBG, 2017) declaran finalmente que, en la medida en que los productos tengan un proceso de producción y desarrollo integrado en diferentes países, ningún país sería autónomo a nivel de comercio internacional, razón por la cual se involucran esfuerzos de distintas compañías de orígenes diversos para administrar o conformar las Cadenas Globales de Suministro (CGS), que se definen como un sistema de gestión del proceso productivo global del producto o servicio. En ese sentido, la presente investigación aborda este análisis desde los conceptos de CGS, para la industria automotriz en Colombia.

Respecto a la competitividad de Colombia en las CGS y la baja inserción del país en ellas, el Ministerio de Comercio, Industria y Turismo en el CONPES de 2019, identifica que es poco probable pretender que se desarrollen en el país, procesos completos de estudio de toda la Cadena de Suministro dentro del territorio nacional; por tanto, prefiere plantear una perspectiva en la que el país y su industria deban enfocarse: Acceder a estas CGS desde un segmento o una porción de ellas. Esta directriz no es ajena a la industria automotriz nacional, ya que Colombia no cuenta con el desarrollo tecnológico e industrial para llevar a cabo un proceso completo a lo largo de esta cadena de suministro (CS). Respondiendo a las exigencias de la actualidad y al contexto del país, y las estrategias que impulsan diversas entidades del Estado como el Ministerio de Comercio, Industria y Turismo de Colombia y Procolombia, van dirigidas a promover el acceso de la industria colombiana a las CGS de los distintos productos y servicios.

De aquí se definió como objetivo del presente artículo, la delimitación de los efectos y las aplicaciones de la administración eficiente de los impuestos, como soporte para el acceso a las CGS, por parte de la industria automotriz colombiana, describiendo la integración horizontal de la CGSA para identificar las actividades relevantes que permitan buscar relaciones fiscales. Es importante la definición de estrategias tributarias desde la industria automotriz colombiana, encaminadas a llegar a ser parte de estos sistemas de gestión y el papel del contador en este sector productivo, para la administración eficiente de impuestos en las CGS. De igual manera, se debe considerar que la profesión contable tiene información transversal de las compañías, la cual suministra herramientas que soportan y contribuyen a crear, participar y gestionar la toma de decisiones estratégicas, analizando la importancia y las diversas problemáticas que se presentan.

Según Shunko, Do \& Tsay, (2016), la investigación tradicional en la literatura sobre operaciones y gestión de la cadena de suministro tiende a recomendar ubicaciones basándose principalmente en los costos diferenciados de producción y la distancia física, ignorando el papel de los impuestos o fiscalidad. Las multinacionales que ubican sus operaciones estratégicamente de sus cadenas de suministro en regiones o lugares con bajos costos impositivos, pueden distribuir las ganancias entre las divisiones y así mejorar las utilidades finales después de impuestos. La adopción de una concepción en la legislación de una jurisdicción trae consecuencias para las operaciones económicas. En ese sentido, el concepto de CGS ha tenido relevancia en el sector empresarial y gubernamental, ya que permite ver un panorama económico global del proceso y considerar cada proveedor y cliente, así como el nivel que cumple dentro de la cadena. "De la misma manera como los productos han evolucionado, la logística en el mundo competitivo está cambiando a unas velocidades sorprendentes, al punto el concepto de logística evolucionó al de administración de cadenas de suministro" (Acero, 2006).

Es necesario también, identificar los principales elementos de cada uno de los cinco factores clave: aduanas, costos, tiempo, almacenaje y transporte, de una cadena global de suministro - CGS, respecto a las variables transporte y costos particularmente, se recomienda una mejora en los servicios que actualmente se ofrecen, dado que el costo está directamente relacionado con el servicio. Por su parte las rutas fiscales establecidas actualmente necesitan una mejora en su infraestructura a su vez, es necesario una planeación respecto a la implementación y mejora de nuevas rutas, para contar con mejores corredores logísticos de vanguardia que efectivamente cubran las necesidades de los mercados internacionales (Zamora y González, 2018).

Según la OCDE (2014), los beneficios de la participación de las CGS son múltiples, pues proporcionan la posibilidad a las empresas de ingresar a los mercados, especializarse 0 desarrollarse en actividades intermedias dentro de una cadena, lo cual les permite a los proveedores sustentar la producción en segmentos de mayor valor de sus industrias, aprender nuevos procesos, cumplir con los estándares, mejorar el acceso 
a nuevos mercados, facilitar las exportaciones e importaciones en el comercio interno de la cadena, fomentar la utilización de las tecnologías de red y generar nuevas fuentes de capital. A nivel jurisdiccional, las CGS, permiten a los países especializarse en áreas de ventaja comparativa, lo que mejora el crecimiento de la productividad y respalda los salarios y los ingresos. Al mismo tiempo ha llevado a una interdependencia e interconexión de las economías.

Una de las decisiones más importantes en la gestión de las Cadenas de Suministro Globales consiste en tomar decisiones acerca de la conveniencia de subcontratar o producir en origen. Esta decisión se toma con referencia a un conjunto de modelos cuantitativos y cualitativos. Sin embargo, con frecuencia se ha pasado por alto un factor clave: las consecuencias fiscales o tributarias que resultan de los precios de transferencia para las empresas multinacionales (Drtina y Correa, 2008). Hoy la operación de la cadena de suministro (SC) se enfrenta a grandes incertidumbres en el lado de la demanda, así como a un complejo proceso de distribución en una geografía dada. Comprender, por parte de los tomadores de decisiones de las interdependencias y procesos de una SC, es esencial para aprovechar las oportunidades y mitigar los riesgos (Giraldo et al., 2019).

\section{MATERIALES Y MÉTODOS}

El acceso a las Cadenas Globales de Suministro (CGS), ha representado un objetivo importante para las organizaciones y empresas a nivel mundial, que como mencionan Jüttner y Maklan (2011), existen factores que fomentan los estudios sobre la resiliencia para desarrollar métodos y sistemas que sean lo suficientemente seguros y flexibles como para reaccionar ante desconexiones inesperadas. El concepto de resiliencia organizacional se ha llevado en la actualidad a las denominadas Cadenas Globales de Suministro [CGS] para describir la necesidad de que las organizaciones reaccionen más rápido a los cambios en el entorno empresarial (Nogueira y Hallal, 2013) y de manera interconectada. Por otro lado, se ha definido la resiliencia como un conjunto de capacidades organizacionales responsables de combatir y superar las interrupciones o desconexiones repentinas e inevitables en el entorno con acciones proactivas y reactivas de anticipación, adaptación, respuesta, recuperación y lecciones aprendidas, siempre interconectadas (Kamalahmadi y Parast, 2016; Ali et al., 2017).

\section{Integración vertical}

El concepto de desagregación capta un aspecto importante de la dinámica de la economía mundial, pero hay otra dimensión crítica de la perspectiva analítica para el desarrollo de las CGS. A comienzos del siglo XX, Henry Ford ideó e implementó un modelo de negocios que apuntaba a integrar varios segmentos (funciones) de un proceso de producción bajo un solo paraguas de capital y administración a través de la adquisición de una variedad de compañías. El modelo, más tarde conocido como una estrategia de integración vertical, se convirtió en un modus operandi en la era de la producción masiva. Los primeros estudios de integración vertical se centraron en las imperfecciones del mercado. Una empresa integra otras entidades para corregir las distorsiones de poder del mercado preexistentes, como la doble marginación, la gratuidad o la ejecución hipotecaria. Otra línea de pensamiento considera la preclusión de los costos de transacción como un motivo principal para la integración vertical, en la cual la internalización de las actividades de producción es una medida para evitar los costos potenciales de establecer relaciones comerciales formales en condiciones de plena competencia (WBG, 2017).

Desde el punto de vista de la economía de costos de transacción, los costos de la producción incluyen no solo los costos directos de escribir, monitorear y hacer cumplir los contratos, sino también las ineficiencias de desempeño causadas por riesgos contractuales dentro de la relación comercial. Uno de los principios básicos de la economía de los costos de transacción es que los contratos son incompletos en cuanto a los términos de intercambio entre las partes, y no pueden ser regulados debido a la asimetría de información. Cuando las partes están encerradas en el costo de transacción, el carácter incompleto de los contratos evoca riesgos contractuales de varios tipos, pero la integración vertical se adelanta a estos riesgos al interiorizar relaciones con el objetivo de unificar las empresas. Entonces, la integración vertical se convierte en el modo preferido de organizar las cadenas de suministro cuando el beneficio de atenuar el comportamiento oportunista de las partes dentro de la relación, supera el costo de asignar ineficazmente recursos asociados con arreglos burocráticos (Daniels et al., 2010).

\section{Cadenas Globales de Suministro}

El término Cadenas de Suministro se concibió en estudios de gestión empresarial. Porter (1998) adaptó el concepto como un marco básico para desarrollar una estrategia corporativa, con el fin de promover la competitividad de la empresa dirigiendo la atención al sistema completo de actividades involucradas en la producción y el consumo de un producto. Una entidad corporativa primero se descompone en un conjunto de 
actividades comerciales con funciones individuales, que constituyen unidades analíticas para diagnosticar la ventaja competitiva de la empresa.

Cuando una compañía tiene una estructura organizacional relativamente atomizada, las tareas de cada unidad, tales como diseño de productos, aprovisionamiento, mercadeo y distribución, tienden a definirse de una manera, que persigue el objetivo individual de esa unidad particular, que puede o no entrar en conflicto con el objetivo de otras unidades y en general de la compañía. Sin embargo, en la perspectiva de la CS, todas las actividades deberían organizarse colectivamente para garantizar el funcionamiento óptimo de la entidad corporativa en su conjunto (Ballesteros y Ballesteros, 2004).

Con este fin, la naturaleza de los vínculos entre las actividades (cadenas de suministro) se examina cuidadosamente, similar a dibujar un gráfico anatómico de una empresa: para identificar externalidades potenciales a través de la coordinación funcional cruzada, que es una fuente importante de la ventaja competitiva de las empresas. Por el contrario, los estudios de CGS se originaron en el área de la sociología (Persson, G., 1997). La administración eficiente de impuestos en la cadena de suministro, o TESCM en su acepción inglesa (Tax Effective Supply Chain Management), tiene como objetivo optimizar el impacto global tributario de cambios en los procesos de negocios actuales y futuros, así como el flujo de mercancías y activos, lo que permite incrementar la rentabilidad y la eficiencia de las cadenas de suministro (Barbosa y Oundjan, 2009).

A diferencia del concepto de cadena de suministro de Porter (1998), que trata principalmente de cómo las estrategias firmes pueden renovarse cambiando el enfoque a la configuración de actividades comerciales, los estudios de CGS consideran la generación y transferencia de insumos dentro del sistema, delimitando fronteras, como consecuencia de los esfuerzos firmes para optimizar las redes de producción y el mecanismo de cómo la estructura de distribución de los insumos afecta la elección de la forma organizativa de las redes internacionales de producción. El análisis de CGS no es una extensión global del enfoque de la cadena de suministro de Porter (1998), porque el alcance y la motivación son diferentes. Así se da la evolución del concepto de CGS, según el informe del WBG (2017) y la definición de Clúster que según Monroy (2016), se define como los beneficios en la aglomeración geográfica. La proximidad entre empresas y el establecimiento de instituciones y procesos de apoyo a las industrias dentro de un área geográfica; algunas de ellas incluyen la cercanía a materias primas y mercados, un suministro constante de mano de obra calificada y el apoyo de instituciones y gobierno.

\section{Planificación colaborativa en las CGS}

En la última década se presenta una variación del concepto de CGS, el cual señala que existen varias formas de planear las decisiones en las CGS y que están relacionadas con la planeación de la estrategia de operaciones, la administración de la demanda, la planeación y programación de la producción, y los esquemas de contratación y mecanismos de distribución. La planificación conjunta es usada para alinear a los miembros y coordinar las decisiones en cuanto a reabastecimiento, inventarios, colocación y entrega de las órdenes, y se basa en el hecho de que los socios colaboradores deben trabajar juntos para resolver los problemas de la CS (Herrera, 2014). En una CGS se puede distinguir dos procesos básicos, como son el proceso de producción y el proceso de distribución. Según Herrera (2014), "El concepto de planificación colaborativa se puede aplicar tanto al proceso de planificación de la distribución de los productos hacia los clientes, como en el proceso de planificación de suministros de los proveedores". Se puede ver que una CGS integrada al flujo de información relevante de la oferta y demanda genera la evolución del concepto en valor agregado desde cada segmento y Tier, o nivel jerárquico de la CGS.

La planificación colaborativa en un contexto de CGS, se centra en la coordinación de la planificación y el control de las operaciones de los distintos miembros de la cadena. Esta se interesa por establecer diferentes procesos de planificación y los procesos de intercambios de información para mejorar las actividades de planificación a lo largo de los niveles jerárquicos o Tier, basados en identificar distintos dominios de planificación locales como partes de una CS y en establecer intercambios de datos entre los distintos dominios, con el fin de mejorar esas planificaciones locales (Herrera, 2014).

\section{Organizaciones multilaterales en las CGS}

En la actualidad no existen indicadores o simuladores para medir estimaciones en el impacto legislativo colombiano, por lo cual solo se toma una pequeña muestra de un grueso de organizaciones internacionales. Al analizar la posición de las organizaciones internacionales que emitan pronunciamientos sobre la aplicación y conformación de CGS, se identifican tres actores internacionales de distintos enfoques en su objeto, pero que tienen importantes aportes sobre el tema de CGS: la Organización para la Cooperación y el Desarrollo Económico, la Organización Mundial de Comercio y el World Bank Group. 
Incorporación de lineamientos de la OCDE en la normativa colombiana: con la Ley 1479 de 2011 se aprueba la "Decisión del Consejo de la Organización para la Cooperación y el Desarrollo Económico (OCDE), que establece un centro de desarrollo de la organización", y avaló el compromiso del Gobierno colombiano en el proceso de ingreso a la OCDE, para lo cual el mismo gobierno entra a participar en el centro de desarrollo, con el fin de: conjugar los conocimientos y la experiencia disponibles en los países participantes, tanto acerca del desarrollo económico como de la formulación y ejecución de políticas económicas de tipo general; adaptar dichos conocimientos y experiencia a las necesidades reales de los países en proceso de desarrollo económico y poner los resultados a disposición de los países en cuestión, utilizando los medio apropiados (Presidencia de la República de Colombia, 2017, Decreto №2120).

Además, la OCDE es la organización encargada de emitir las directrices de las normas de precios de transferencia. Con la Ley 1819 del 29 de diciembre de 2016, modificada por el Decreto 2120 del 15 de diciembre de 2017, el Gobierno colombiano establece adoptar un enfoque estandarizado en tres niveles de la documentación sobre precios de transferencia, que harán parte de la información comprobatoria de precios de transferencia, el informe Local, el informe Maestro y el informe País por País, además de definir quiénes serán los responsables de presentar esta información. Entre otras, mediante la Ley 1661 de 2013 se aprobó la asistencia administrativa mutua en materia fiscal por parte de la OCDE. Dicha asistencia incluye intercambio de información fiscal para ser auditada, asistencia en el cobro de los impuestos de renta y patrimonio y asesoría en establecimiento de medidas cautelares. Son importantes las implicaciones normativas del país por cuenta de esta organización.

A diferencia de la OCDE, la OMC es una organización que enfoca sus pronunciamientos y foros sobre las CGS, con el objeto de colocarlas en el foco del debate. Luego de hacer una revisión minuciosa, no se encuentra regulación al respecto por parte de la OMC sobre las CGS; en otras palabras, no ha logrado capturarlas en alguna norma, lo cual se debe a la complejidad de estas estructuras de comercio y sus constantes cambios en sus esquemas de comercio, propios de estas cadenas globales.

En un enfoque distinto, la OMC ha emitido normas que le atañen de manera indirecta a estas CGS, como lo es el caso del Acuerdo General sobre Aranceles Aduaneros y Comercio de 1994 (GATT). El artículo VII de este Acuerdo trata básicamente de las normas de valoración que debe sostener un país para el libre flujo de mercancías. El Acuerdo de Valoración específicamente busca ser la primera base para determinar el valor de aduana de las mercancías, para así ajustar los precios según la valoración de cada caso específico; para una CGS constituye una de las primeras líneas de restricción en la logística de mercancías, de la cual se debe tener constante control y medición, además de una adecuada planeación.

Para Colombia, este impacto aduanero se evidencia al adoptar estas disposiciones normativas de la OMC, lo cual abrió el comercio nacional; sin embargo, para esta investigación la gestión de las administradoras aduaneras del país constituye un punto para mejorar desde su legislatura, buscando impactar de forma positiva la competitividad del país, por ser la puerta de acceso al flujo de mercancías que estas CGS demandan y proporcionan. El Grupo del Banco Mundial (WBG); el Grupo del Banco Mundial es una institución de desarrollo internacional, fuente de asistencia financiera y técnica para países en desarrollo, fundado en 1944 y establecido por los acuerdos adoptados por sus 189 países miembros. Esta institución ha desarrollado detallados reportes anuales de la evolución de las CGS. El reporte WBG, de 2017, pone especial atención al impacto de desarrollo económico propiciado por las CGS, para los países en vías de desarrollo.

En una revisión de la aplicación y conformación de las CGS, además de lo sugerido por la OCDE, el WBG propone una inclusión de los países en desarrollo a los niveles intermedios de las CGS, planteando una redistribución de valor generado por nuevas oportunidades de negocio en estos niveles intermedios. Sostiene que los servicios de una CGS toman especial importancia, pues el WBG (2017) afirma que los servicios constituyen los enlaces vitales de conexión a CGS, así como una gama de insumos obtenidos en condiciones de plena competencia. Estas CS son globales no solo cuando las transacciones cruzan fronteras, sino también cuando los consumidores o proveedores se dedican a establecer una presencia comercial en el extranjero (WBG, 2017). Dado que una gran parte del comercio de bienes incluye el comercio de los servicios, el desarrollo del sector de servicios nacionales y el acceso a insumos de servicios importados, pueden influir en la ventaja comparativa del país y también expone una tendencia creciente de los acuerdos especiales que las jurisdicciones otorgan a CGS de determinados sectores, o bien sean grandes marcas para atraer su inversión, eliminando barreras proteccionistas o concediendo tarifas especiales o nulas de impuestos.

Para Colombia, la posición del WBG tiene un impacto considerable. Al pertenecer a este grupo desde 1946, el país es uno de sus principales objetos de estudio en sus indicadores de desarrollo, al posicionarlo dentro de los cinco países que dirigen estrategias concretas a nivel normativo para el acceso a CGS, especialmente al sector automotor, junto con Brasil, Francia, Marruecos y Uruguay (WBG, 2017). Estas normas comprenden 
esfuerzos del Ministerio de Comercio, Industria y Turismo, principalmente con la creación del Programa de Transformación Productiva, el cual promueve iniciativas nacionales en coordinación con las comisiones de competitividad regional, evaluadas por el Departamento de Planeación Nacional.

\section{Integración horizontal de la cadena global de suministro automotriz}

La cadena global de suministro automotriz (CGSA) es una de las cadenas más complejas, pues se requieren más de quince mil partes para conformar un vehículo y más de 40 tipos de servicios que son propios de la industria, que se necesitan para llevar a cabo el ciclo completo de la CGSA. Definir de forma completa la CGSA es una labor demasiado extensa, por este nivel de complejidad; como respuesta, se hace un análisis horizontal que permita simplificar en las actividades del core business del negocio automotor. Es importante resaltar que todo el proceso demanda la participación de más de una jurisdicción, y siempre esta ejecución se soporta mediante el negocio internacional por excelencia al exigir siempre dos elementos: calidad y costos bajos (Institute of Management Accountants, 1996), los cuales son la vía de acceso a estas CGSA para la industria automotriz colombiana.

El acceso debe estar focalizado al Tier o nivel jerárquico objetivo, dentro de la CGSA, el cual depende de la especialización que tengan las empresas. De acuerdo con lo anterior, se plantean cuatro grupos de actividades esenciales para definir una cadena global de suministro automotriz, las cuales se ordenan en: planeación, acuerdos, producción y entrega, respectivamente (Osorio, 2017).

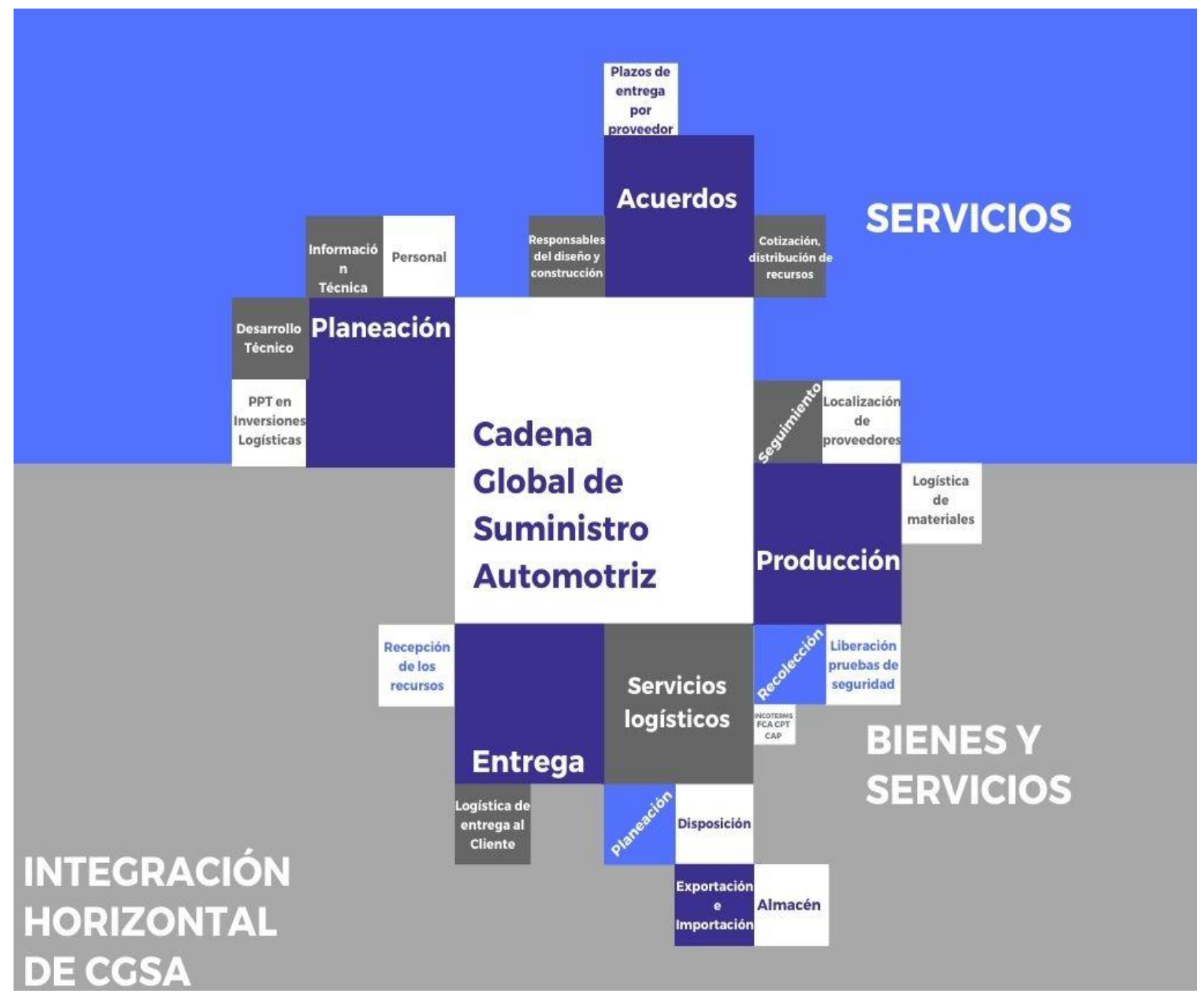

Fig. 1: Cadena global de suministro automotriz (CGSA)

\section{Actividades externas a las CGS}

Todas las CGS, encuentran la necesidad de mantenerse en la mejora y crecimiento continuo, pues en un mundo competitivo "el que no crece simplemente decrece" (Gallardo, 2012). Por tal razón, se designan estrategias continuas que demandan productos y servicios adicionales. Estas estrategias de mejora se 
pueden resumir en los siguientes aspectos: la creación de mecanismos para optimizar los procesos internos de cada grupo de actividades, generar impulso a nuevos proveedores, promover el desarrollo de los proveedores (escalar en los Tier o niveles), crear nuevas plantas de producción en distintas jurisdicciones y generar estrategias constantes de sostenibilidad promoviendo la economía circular, el desarrollo del ecosistema productivo de manera sustentable y buscar ahorros ambientales. Además, y fuera de las actividades mencionadas, para las cadenas del sector automotor existen certificaciones especiales para su acreditación. Estas certificaciones son emitidas por entidades como la Asociación de Fabricantes de Automóviles y Equipos Originales (OEM, por su sigla en inglés) con la ISO/TS/6949 y entes gubernamentales como la Asociación Estratégica Aduana-Industria Contra Terrorismo (C-TPAT, por su sigla en inglés) (Osorio, 2017).

\section{RESULTADOS Y DISCUSIÓN}

Luego de describir las actividades del core business en el análisis horizontal anterior, se pueden identificar actividades de servicios y de proveeduría específicas del sector, así como el ejercicio logístico de importaciones y exportaciones. Para poder delimitar los efectos y las aplicaciones de una administración eficiente de los impuestos en la Cadenas Globales de Suministro Automotriz [CGSA], es preciso tener presente que la complejidad del sistema tributario colombiano es importante, por lo cual se buscan simplemente los elementos comunes de comercio dentro de los grupos de actividades mencionados, que confluyen en: la exportación de servicios, la exportación de bienes (materiales, autopartes o componentes) y la importación de bienes (vehículos, partes o componentes), todos a la luz de la industria colombiana automotriz.

Los retos impuestos por las nuevas formas de hacer negocios, motivan el surgimiento de otras perspectivas estratégicas, como en el caso del enfoque sustentable, que surge por el compromiso de impulsar el desarrollo económico y social, sin poner en riesgo los recursos naturales que proveen las materias primas para el sector productivo a nivel mundial (Ramírez et al., 2019). Los hallazgos realizados en estudios previos son coherentes con Heredia et al. (2019), los gerentes latinoamericanos deben desarrollar la capacidad de combinar exploración y explotación, porque las empresas ambidiestras tienden a superar a otras en un entorno incierto. De hecho, estos autores recomendaron combinar recursos internos y externos en un entorno de baja calidad institucional; mientras tanto, en un entorno institucional positivo, las empresas pueden ser ambidiestras sin una mayor dependencia de la cooperación externa (Acevedo y Díaz, 2019).

\section{Exportación de servicios}

El impuesto sobre las ventas; existen más de 40 tipos de servicios en las diferentes etapas de la cadena (planeación, acuerdos, producción y entrega). Para verlos desde un punto de vista fiscal, toma relevancia el impuesto sobre las ventas aplicado a las exportaciones de servicios. A continuación, se describen las cualidades fiscales exigidas a los exportadores de servicios. El artículo 481, literal c) ET, denomina exportación de servicios a aquellos que son prestados dentro del territorio nacional, pero que son aprovechados entera o exclusivamente en el exterior, por empresas o personas sin negocio o actividades en Colombia. Las prestaciones de estos servicios adquirirán la calidad de exentos, indicando que son gravados, pero a tarifa cero.

Para el caso de los exportadores (bienes o servicios), en Colombia la legislación prevé la posibilidad de solicitar devolución o compensación únicamente por el Impuesto Sobre las Ventas (IVA), descontable asociado directamente con la producción de bienes o servicios exportados. Lo anterior, aplicaría a partir del momento en el que la compañía inicie con la exportación de servicios y cumpla los demás requisitos establecidos por la ley. Podrán solicitar devolución o compensación de saldos a favor originados en las declaraciones del impuesto sobre las ventas, los responsables pertenecientes al régimen común de los bienes y servicios relacionados como exentos y otros casos puntuales, además de cumplir con algunas formalidades.

Para efectos fiscales, debe existir relación de causalidad directa entre los conceptos que dan lugar a saldos a favor y la actividad comercial desarrollada, para que se configure la viabilidad de devolución o compensación del IVA, conforme lo expone nuestra legislación fiscal. Seguidamente se enlistan aquellos que podrán solicitar devolución o compensación del IVA. Los prestadores de servicios que desarrollen sus actividades en el país, pero que se utilicen exclusivamente en el exterior, servicios exentos del IVA, conforme lo expone el literal c) del artículo 481 del Estatuto Tributario (ET). Quienes hayan sido objeto de retención del IVA, hasta la concurrencia del saldo a favor originado en las retenciones que les hubieren practicado y que hayan incluido en las declaraciones del periodo correspondiente, artículo 850, parágrafo 1, ET (Estatuto Tributario). También podrán solicitar devolución o compensación de saldos a favor originados en las declaraciones del IVA, quienes sean productores de bienes gravados a la tarifa del cinco por ciento $(5 \%)$ o prestadores de servicios gravados a la tarifa del cinco por ciento (5\%) tipificados en los artículos 468-1 y 468-3 ET, respectivamente. Esta 
devolución aplicará solo para los excesos de IVA pagado en relación directa con la operación que da lugar a la cualidad.

Cuando los responsables que tratan los artículos 477 y 481 ET (Estatuto Tributario), que adicionalmente realicen operaciones gravadas o excluidas, la devolución o compensación solo procederá por el IVA exento descontable asociado directamente con la producción de bienes o servicios exportados o exentos, de conformidad con lo dispuesto con el artículo $490 \mathrm{ET}$, el cual menciona la proporcionalidad para efecto del cálculo del saldo por imputar. Estas cualidades tienen origen en la normativa colombiana, con el Decreto 2277 de 2012, con el cual se reglamenta parcialmente el procedimiento de gestión de las devoluciones o compensaciones. Este fue modificado por el Decreto 2877 del 11 de diciembre de 2013, que dio lugar a la conformación normativa actual de la legislación colombiana.

En el momento en que una empresa desarrolle conjuntamente operaciones gravadas excluidas o exentas, en un mismo periodo y el desarrollo conlleve generar impuestos descontables, estos se imputarán proporcionalmente; para ello se deberá seguir lo expuesto en el artículo 490 del ET. Lo anterior corresponde a las cualidades de las operaciones. Ahora bien, con la expedición del Decreto 2223 de 2013 se reglamentó parcialmente el artículo 481 del ET; en este, se exponen los requisitos formales que acreditarán la exención del IVA por la exportación de servicios, los cuales se listan a continuación.

\section{Efectos tributarios de los impuestos de renta pagados en el exterior}

Para la norma colombiana es posible descontar los impuestos de renta pagados en el exterior directamente en la tarifa. El fin es evitar la doble tributación sobre las mismas rentas; no obstante, el valor de este descuento no podrá exceder el monto de impuesto sobre la renta y complementarios, que deba pagar el contribuyente en Colombia por estos mismos réditos. En atención a la ley 1943 de 2018 se elimina el límite de tiempo para ser tratado como descuento en el tributo, con la única limitante del impuesto sobre la renta y complementarios generado en Colombia sobre las rentas de fuente extranjera de igual naturaleza a las que dieron origen a dicho descuento. Es importante aclarar que la ley 1943 fue declarada inexequible en el año 2019, por lo cual esto aplica solo para las operaciones realizadas en el año de vigencia y en caso de no aprobarse el texto del proyecto de ley 278 de 2019. Para tener derecho a este descuento el contribuyente debe probar el pago en cada jurisdicción, aportando el certificado fiscal de pago del impuesto expedido por la autoridad tributaria respectiva o en su defecto con prueba idónea (Presidencia de la República de Colombia, 1989, ET, literal f, artículo 254).

\section{Descuento de Impuesto al Valor Agregado pagado en la importación}

En el artículo 83 de la ley 1943 de 2018, incluye la opción de llevarse como descuento tributario en las declaraciones de IVA y Renta, los impuestos pagados de IVA, en la adquisición, conformación, importación o construcción de activos fijos reales productivos antes llamados bienes de capital, lo cual genera importantes oportunidades en los costos repercutidos de estos activos, para los consumidores finales y para la vigencia fiscal de 2019. Para los años 2020 y posteriores se sujeta a lo aprobado en el proyecto de ley 278 de 2019 que incluye la posibilidad de llevar este descuento en el impuesto sobre la renta y no sobre las declaraciones de IVA únicamente hasta el año 2022, estas implicaciones impactan significativamente la planeación de CAPEX dispuesta por las compañías del sector automotriz en Colombia dado su plan de inversiones en esta clasificación de activos fijos reales productivos.

\section{Exportación e importación de bienes}

Los INCOTERM en la CGSA; un INCOTERM es un término contractual pactado, que define las obligaciones mercantiles en las operaciones de comercio internacional sobre la mercancía objeto de la negociación. Los resultados dependen del tipo de INCOTERM y del país origen donde se negocie. Definir un INCOTERM ya no es un tema exclusivo del negocio, también se debe observar desde un enfoque tributario. Como se mencionó en el Capítulo III, los INCOTERM más comunes en la etapa de entrega de la CGSA son el FCA (libre transportista - lugar de destino convenido), CPT (lugar de destino convenido) y CIP (transporte y seguro pago hasta lugar de destino convenido). Se vuelve necesario predecir los costos tributarios que estos deriven y la rentabilidad de la operación durante las CGS, completas no solo enfocándose a un ahorro tributario específico (Carpio, 2012).

Es común que las empresas que negocien este tipo de compraventas, según Bermeo, Lizarazo, Villamil y Vargas (2005), cometan el error de asumir que en el término del INCOTERM se regulan todas las obligaciones que se desean incluir en un contrato o, por el contrario, que el INCOTERM que se pacta en un contrato no refleje las verdaderas condiciones de negociación. Por tanto, la presente investigación considera que sucede igual al no percatarse de los efectos tributarios que implican, y mucho menos quedar reflejados en estos 
contratos de negociación. Esto conlleva a incurrir en costos fiscales no presupuestados dentro de las actividades de planeación de la CGSA y a equiparar estas cargas económicas entre las partes que les corresponda asumir estos impuestos. Hay que considerar en este sentido la descripción de cada uno de los diferentes procesos inherentes a la cadena de suministro, que permite entender las interrelaciones que se presentan entre ellos. Este conocimiento de la estructura de la cadena es de vital importancia en cualquier organización para poder mantenerse en un entorno globalizado, ya que permite el desarrollo de estrategias de diferenciación con respecto a sus competidores (De La Hoz et al., 2019).

En Colombia definir el valor de aduana, siempre incluirá los fletes y seguros más el valor FCA (libre transportista - lugar de destino convenido) de las mercancías. Esto está alineado con el Acuerdo General sobre Aranceles Aduaneros y Comercio de 1994 - GATT (OMC, 1994). Es decir, la medición de la base gravable de los impuestos arancelarios siempre reflejará un INCOTERM CIP (transporte y seguro pago hasta lugar de destino convenido) desde la mirada de un término FCA. Los INCOTERM en materia de IVA; el artículo 420 del Estatuto Tributario Nacional menciona que dentro de los hechos generadores del IVA hay que considerar tanto la importación como la venta para determinar los efectos tributarios de los INCOTERM, el IVA en la venta de los bienes corporales muebles, entendida esta última como la transferencia de dominio sobre los bienes corporales muebles a cualquier título. El IVA se causa al momento de la entrega o al momento de la expedición de la respectiva factura, lo que suceda primero, y todas las demás que refiere el artículo 429 del ET (Presidencia de la República de Colombia, 1989, ET, artículo 429).

Los INCOTERM en materia de Impuesto sobre la Renta; los INCOTERM regulan lo referente a los riesgos y las obligaciones que se asumen en una negociación. Estos no contemplan el traspaso de la propiedad de los bienes dentro de los Tier (por ejemplo, el proveedor del proveedor sería tier 2, el proveedor es tier 1 y así sucesivamente) o niveles de la CGSA. El régimen tributario colombiano determina en el artículo 24 del Estatuto Tributario, que "constituyen ingresos de fuente nacional los obtenidos en la enajenación de bienes materiales, a cualquier título, que se encuentren dentro del país al momento de su enajenación". Es decir, que independiente de que se pacte un INCOTERM, la enajenación o transmisión de la propiedad no se entendería realizada en el territorio nacional y por consiguiente no es un ingreso de fuente nacional para el proveedor del exterior y el pago se hará sin retención en la fuente de renta en Colombia (DIAN, 2005, Concepto 071190 del 30 de septiembre). No atender la regulación cambiaria colombiana puede desencadenar temas tributarios; para el país, la omisión de las normas cambiarias soporta el rechazo de la deducción del costo o gasto en la base gravable del impuesto sobre la renta. Utilizar los canales cambiarios adecuados con una concordancia en las declaraciones de importación, según el INCOTERM pactado, es lo que debe vigilar la entidad declarante al momento de importar bienes corporales muebles provenientes de estas CGSA.

El C-TPAT y la valoración aduanera; la adopción de este tipo de certificación, el Customs Trade Partnership Against Terrorism (C-TPAT, por su sigla en inglés), no solo trae beneficios logísticos como la reducción en el número de inspecciones, el acceso a carriles exclusivos terrestres en las fronteras con Estados Unidos y la excepción de exámenes estratificados, sino que además reduce un $2 \%$ de los impuestos aduaneros. Así mismo, la agilidad en el despacho aduanero permite menores costos por el uso del suelo aduanero y se evita la pérdida de los excedentes en reconocimiento de aduanas simplemente pagando las contribuciones (Osorio, 2017). En general es de alto impacto con las relaciones mercantiles entre la aduana y la empresa privada, lo cual disminuye la contribución directamente, beneficia los costos aduaneros y mejora la competitividad, para todos los productos que, además de ser parte de la CGSA, tengan destino final territorio estadounidense.

El objetivo de las normas de valoración aduanera es determinar el valor de aduana de los bienes corporales muebles; por tanto, determina la base gravable para efectos de los tributos aduaneros. Es importante señalar cómo el Estatuto Tributario refiere la base gravable del IVA en las importaciones: el artículo 459 (ET) indica que: La base gravable, sobre la cual se liquida el impuesto sobre las ventas en el caso de las mercancías importadas, será la misma que se tiene en cuenta para liquidar los derechos de aduana, adicionados con el valor de este gravamen. El parágrafo menciona que Cuando se trate de mercancías cuyo valor involucre la prestación de un servicio o resulte incrementado por la inclusión del valor de un intangible, la base gravable será determinada aplicando las normas sobre valoración aduanera, de conformidad con lo establecido por el acuerdo de valoración de la Organización Mundial de Comercio, OMC.

La base gravable sobre la cual se liquida el impuesto sobre las ventas -IVA en la importación de productos terminados producidos en el exterior o en zona franca con componentes nacionales exportados, será la establecida en el inciso primero de este artículo adicionado el valor de los costos de producción y sin descontar el valor del componente nacional exportado. Esto requiere reflexionar los momentos en los cuales estos servicios incluidos dentro del valor de aduana causan IVA y retenciones en la fuente, por entenderse de fuente nacional o extranjera. 
La normatividad aduanera contempla la contingencia que un usuario aduanero pueda declarar el valor de aduana de manera provisional en algunas circunstancias; es decir, que se presente la declaración de importación con valores que luego pueden ser modificados y subsiguientemente pagar un mayor valor de impuesto sin lugar a sanciones. Frente a esta opción el Estatuto Tributario nacional señala en su artículo 429, parágrafo, que "cuando el valor convenido sufriere un aumento con posterioridad a la venta, se generará el impuesto sobre ese mayor valor, en la fecha en que este se devengue", para detallar la importancia en el evento en el que al cambiar estos valores iniciales, se haga necesario pagar también un mayor IVA; o sea, alinear completamente el valor de aduana y los tributos aduaneros pagados con el IVA generado a las importaciones.

Evitar la doble tributación internacional; en Colombia existen 11 convenios de doble imposición (CDI), que le atañen a las CGSA, y los países involucrados son: Italia, España, Chile, Suiza, Canadá, México, República de Corea, India, República Checa, Portugal y Venezuela. Definir la doble imposición inicialmente se refiere a gravar un impuesto idéntico o similar en dos o más estados diferentes, suponiendo que el hecho imponible sea el mismo y por igual periodo (Bermeo et al., 2005). Los CDI son acuerdos que suscriben los estados para que las empresas beneficiadas pueden llegar a tener altos réditos para las CGSA, si se planean de manera fiscalmente eficiente, pues actúa como un escudo a la doble tributación y también como una eficiencia tributaria al reducir la carga impositiva total (Carpio, 2012).

Precios de transferencia en las CGSA; los precios de transferencia son los precios a los que las compañías vinculadas deben transar sus bienes tangibles e intangibles, comparten recursos o prestan servicios entre sí. En el momento en que las operaciones tienen lugar en más de dos jurisdicciones, las compañías vinculadas pueden evitar el impuesto de renta mediante la administración de dichos precios de transferencia (Torre, 2010).

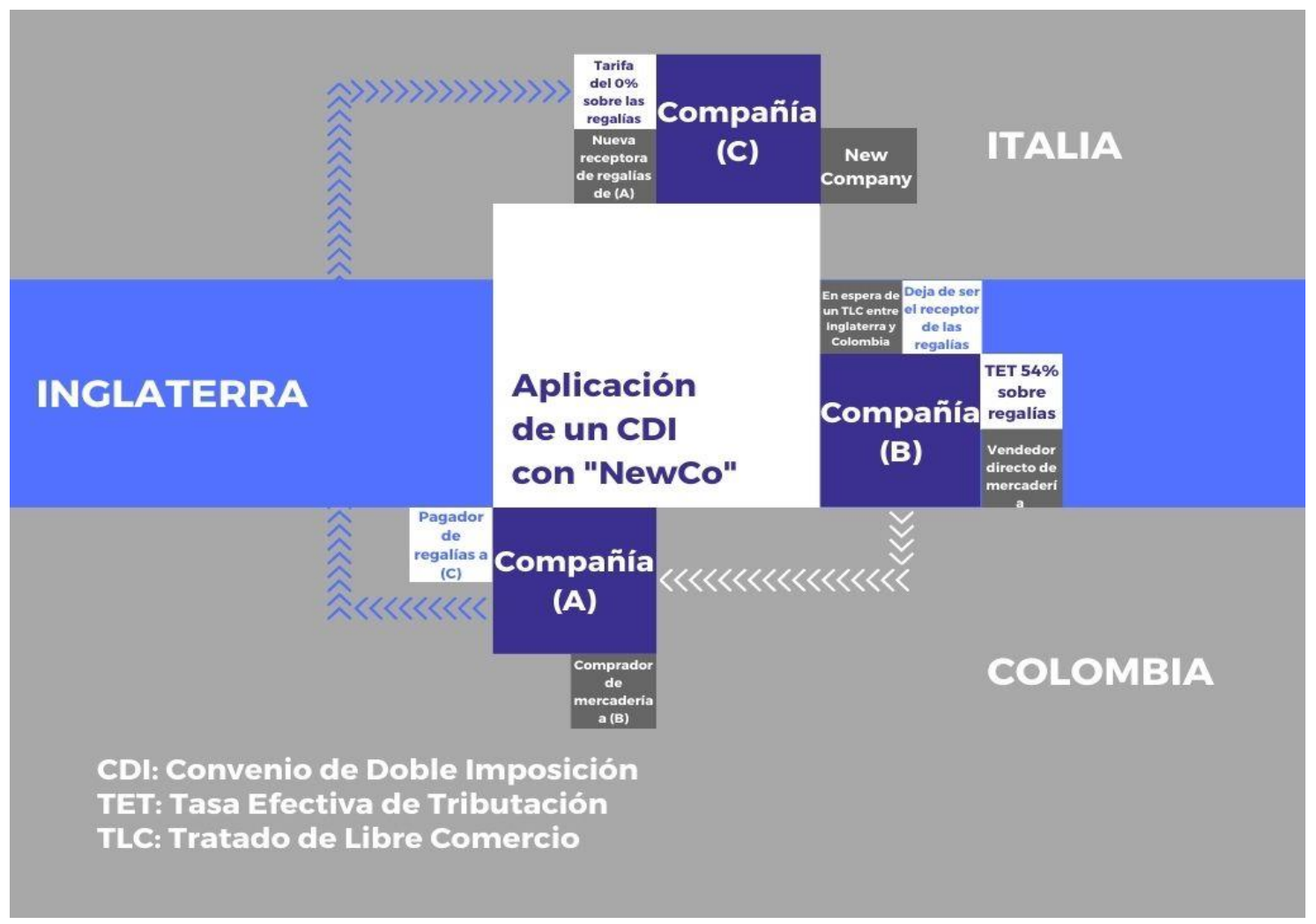

Fig. 2: Aplicación de un CDI con "NewCo"

Cuando Henry Ford ideó e implementó un modelo de negocios que apuntaba a integrar varios segmentos (funciones) de un proceso de producción bajo un solo 'paraguas' de capital, se denominó integración vertical de la cadena de suministro. Los países empezaron a regular esta integración vertical con el objetivo principal de evitar el abuso del principio de plena competencia, pues no en todos los niveles ni en todos los procesos de esta integración vertical, se distribuye de forma equitativa la riqueza generada a lo largo de la CS. Esto obligó a las jurisdicciones a regular este modus operandi, a través de las directrices emitidas por la OCDE sobre precios de transferencia, con el principio rector de la plena competencia.

Para una industria automotriz como la colombiana, el impacto de estas normas de precios de transferencia es muy interesante, ya que el valor generado que representa esta industria dentro de la CGSA es 
relativamente bajo; no obstante, la corrección de precios de estas transacciones entre compañías que sean vinculadas propenderá a no dejar el desequilibrio y redistribución en alguna medida del valor generado a lo largo de la cadena en los procesos desarrollados en el país.

\section{El contexto nacional tributario}

Para ejemplificar el contexto tributario del país, no hace falta mencionar que se considera como uno de los más complejos, no solo por los aspectos técnicos sino por el involucramiento de intereses diversos al momento de legislar sus normas. En la actualidad los intereses se enmarcan en soluciones legislativas a las coyunturas económicas, sociales y políticas por las que el país se enfrenta, resultado de ello se declaró inexequible la ley 1943 de 2018 y se debate el futuro de un nuevo proyecto de ley que sugiere los siguientes cambios normativos que impactan de manera significativa la industria automotriz y su efecto en la vinculación con las cadenas globales de suministro.

Tabla 1: Cambios normativos propuestos para la industria automotriz en Colombia (Fuente: Elaboración propia con datos tomados de DIAN, 2014)

\begin{tabular}{|c|c|c|c|}
\hline Tema & Subtema & $\begin{array}{l}\text { Art. } \\
\text { ET/Otros }\end{array}$ & Principal cambio propuesto \\
\hline IVA & $\begin{array}{l}\text { Base gravable en } \\
\text { importación de } \\
\text { bienes (zonas } \\
\text { francas) }\end{array}$ & 459 & $\begin{array}{l}\text { En la base gravable del IVA en las importaciones, se especifica que } \\
\text { para productos hechos en zona franca con componentes } 100 \% \\
\text { nacionales o nacionalizados, deberán ser facturados por el usuario } \\
\text { de zona franca e ingresar al territorio con el documento que } \\
\text { establezca el gobierno nacional. }\end{array}$ \\
\hline IVA & Bienes exentos & 477 & $\begin{array}{l}\text { Se precisa que el beneficio para vehículos automotores de transporte } \\
\text { público de pasajeros o de servicio público o particular, tendrá } \\
\text { vigencia de cinco (5) años a partir de la entrada en vigencia de la } \\
\text { Ley. }\end{array}$ \\
\hline IVA & Retención & $437-1$ & $\begin{array}{l}\text { Se faculta al Gobierno para disminuir la tarifa de retención en la } \\
\text { fuente para responsables de IVA, que en los últimos } \\
6 \text { periodos generen saldo a favor en sus declaraciones. }\end{array}$ \\
\hline $\begin{array}{l}\text { Mega- } \\
\text { Inversione } \\
\text { s }\end{array}$ & Mega-Inversiones & $235-3$ & Elimina el régimen de las Mega-Inversiones. \\
\hline Patrimonio & Sujetos pasivos & $292-2$ & $\begin{array}{l}\text { 1. Amplía el impuesto al patrimonio hasta el año } 2025 \text {. } \\
2 \text {. Establece que no son sujetos pasivos las sociedades extranjeras } \\
\text { no declarantes de renta en el país y que suscriban contratos de } \\
\text { arrendamiento financiero con residentes en Colombia. } \\
\text { 3. Incluye como sujeto pasivo a las sociedades extranjeras } \\
\text { respecto de su riqueza poseída en el país, } \\
\text { salvo las excepciones contenidas en tratados } \\
\text { internacionales. }\end{array}$ \\
\hline Patrimonio & $\begin{array}{l}\text { Sujetos pasivos, } \\
\text { hecho generador, } \\
\text { base gravable, } \\
\text { tarifa, causación, } \\
\text { etc. }\end{array}$ & $\begin{array}{l}292-2 \\
294-2 \\
295-2 \\
296-2 \\
297-2 \\
298\end{array}$ & Se elimina el impuesto al patrimonio. \\
\hline $\begin{array}{l}\text { Renta } \\
\text { General }\end{array}$ & $\begin{array}{l}\text { Descuento IVA } \\
\text { bienes de capital }\end{array}$ & $258-1$ & $\begin{array}{l}\text { Elimina el artículo } 75 \text { del proyecto de ley que establece el descuento } \\
\text { en renta del IVA pagado por la adquisición, construcción, o formación } \\
\text { e importación de activos fijos reales productivos. }\end{array}$ \\
\hline $\begin{array}{l}\text { Renta } \\
\text { General }\end{array}$ & $\begin{array}{l}\text { Descuento IVA } \\
\text { bienes de capital }\end{array}$ & $258-1$ & $\begin{array}{l}\text { Hasta el año } 2022 \text { se aplicará el descuento del IVA pagado por la } \\
\text { adquisición, construcción, o formación e importación de activos fijos } \\
\text { reales productivos. }\end{array}$ \\
\hline $\begin{array}{l}\text { Renta } \\
\text { General }\end{array}$ & $\begin{array}{l}\text { Eliminación de } \\
\text { descuentos }\end{array}$ & $259-2$ & $\begin{array}{l}\text { 1. Elimina a partir de } 2020 \text { y no de } 2019 \text {, los descuentos tributarios } \\
\text { en el impuesto sobre la renta. } \\
\text { 2. Elimina el descuento de becas por impuestos. }\end{array}$ \\
\hline $\begin{array}{l}\text { Renta } \\
\text { Personas } \\
\text { Jurídicas }\end{array}$ & Descuento ICA & 115 & $\begin{array}{l}\text { 1. Permite deducir el impuesto al patrimonio. } \\
\text { 2. Establece que todo el ICA, avisos y tableros (no el } 50 \% \text { ) será } \\
\text { deducible (no descontable). } \\
\text { 3. Dispone que las regalías no podrán ser tratadas como costo, ni } \\
\text { podrán ser deducibles ni descontables. }\end{array}$ \\
\hline $\begin{array}{l}\text { Renta } \\
\text { Personas } \\
\text { Jurídicas }\end{array}$ & $\begin{array}{l}\text { Descuento } \\
\text { tributario }\end{array}$ & $258-2$ & $\begin{array}{l}\text { Las empresas tendrán un descuento sobre la tarifa de renta que le } \\
\text { corresponda según el art. } 240 \text { del ET (tarifa general para personas } \\
\text { jurídicas), que se aplicará conforme al valor agregado generado a } \\
\text { su producto final, de acuerdo con una fórmula que considera el total } \\
\text { de gastos sin materia prima y la materia prima. }\end{array}$ \\
\hline
\end{tabular}


Tabla 1: continuación.

\begin{tabular}{|c|c|c|c|}
\hline Tema & Subtema & $\begin{array}{l}\text { Art. } \\
\text { ET/Otros }\end{array}$ & Principal cambio propuesto \\
\hline $\begin{array}{l}\text { Renta } \\
\text { Personas } \\
\text { Jurídicas }\end{array}$ & $\begin{array}{l}\text { Ingresos que no } \\
\text { se consideran de } \\
\text { fuente nacional }\end{array}$ & 25 & $\begin{array}{l}\text { Frente a los ingresos de fuente nacional obtenidos en la enajenación } \\
\text { de mercancías provenientes de una sociedad extranjera o sin } \\
\text { residencia, introducidas al país mediante centros logísticos: } \\
\text { 1. Se eliminan los centros logísticos ubicados en } \\
\text { "aeropuertos internacionales" (de los departamentos a los que se } \\
\text { refiere la norma). } \\
\text { 2. Se incluye al Departamento del Chocó. }\end{array}$ \\
\hline $\begin{array}{l}\text { Renta } \\
\text { Personas } \\
\text { Jurídicas }\end{array}$ & Presuntiva & 188 & Establece la renta presuntiva en $3.5 \%$ \\
\hline $\begin{array}{l}\text { Renta } \\
\text { Personas } \\
\text { Jurídicas }\end{array}$ & Tarifa & 240 & $\begin{array}{l}\text { 1. Modifica la estructura y tarifa del impuesto sobre la renta para } \\
\text { personas jurídicas, fijando tarifas diferenciales de acuerdo } \\
\text { con el tipo de empresa: } \\
\text { a. Micro: } 27 \% \\
\text { b. Pequeñas: } 28 \% \\
\text { c. Medianas: } 29 \% \\
\text { d. Grandes: } 30 \% \text {. } \\
\text { 2. Crea un descuento tributario para empresas existentes o que se } \\
\text { creen el territorio nacional, según el valor agregado a su producto } \\
\text { final (con una fórmula). }\end{array}$ \\
\hline $\begin{array}{l}\text { Renta } \\
\text { Personas } \\
\text { Jurídicas }\end{array}$ & Tarifa & 240 & $\begin{array}{l}\text { 1. Se crea una única tarifa general de renta para personas jurídicas } \\
\text { del } 32 \% \text { (se elimina la disminución gradual de la tarifa para los } \\
\text { años } 2020,2021 \text { y 2022). } \\
\text { 2. Se elimina la tarifa de } 9 \% \text { para hoteles, parques temáticos y } \\
\text { muelles náuticos. } \\
\text { 3. Se elimina la sobretasa en el impuesto sobre la renta a los } \\
\text { servicios financieros. }\end{array}$ \\
\hline $\begin{array}{l}\text { Renta } \\
\text { Personas } \\
\text { Jurídicas }\end{array}$ & Tarifa & 240 & $\begin{array}{l}\text { Modifica la estructura y tarifa del impuesto sobre la renta para } \\
\text { personas jurídicas, fijando tarifas diferenciales de acuerdo con el tipo } \\
\text { de empresa: } \\
\text { 1.Micro: } 27 \% \\
\text { 2.Pequeñas: } 28 \% \\
\text { 3.Medianas: } 29 \% \\
\text { 4.Grandes: } 30 \%\end{array}$ \\
\hline $\begin{array}{l}\text { Renta } \\
\text { Personas } \\
\text { Jurídicas }\end{array}$ & Presuntiva & 188 & $\begin{array}{l}\text { Se reduce el porcentaje de la renta presuntiva para el año gravable } \\
2020 \text { del } 1.5 \% \text { al } 0.5 \% \text {. En el } 2021 \text { se mantiene } \\
\text { en el } 0 \% \text {. }\end{array}$ \\
\hline
\end{tabular}

\section{El rol del Contador Público en las CGS}

La Contaduría Pública ha experimentado cambios importantes durante los últimos años, como la necesidad de apostarle a una interdisciplinariedad en lo que apuntan los currículos. No obstante, la vida laboral se resiste a considerar al contable como un gestor dentro de las organizaciones, para ver la profesión con un alto sentido de responsabilidad y como un elemento de desarrollo continuo para las organizaciones; por eso, pocas profesiones son tan elementales en estos sentidos. El perfil del contador gerencial es cada vez más acertado, si se tiene en cuenta la transversalidad de la información que maneja desde su profesión. Hacer uso responsable y ético de esta información es hoy una oportunidad para el contador gerencial.

Las competencias del Contador Público; un factor determinante en los cambios a los que se expone la profesión tiene que ver con las tecnologías emergentes. Los profesionales de la Contaduría Pública deben abrazar este tipo de oportunidades, pues este aspecto asegurará estar activos en el entorno de los negocios (International Federation of Accountants - IFAC, 2017). En una aproximación, al describir las competencias necesarias dentro de este entorno automotor para la profesión, se encuentran el emitir la información financiera, analizar los costos de una CS, estudiar los términos contractuales de las negociaciones para determinar los importes de las transacciones, examinar inversiones, verificar el cumplimiento normativo y analizar coberturas, términos de financiación y participación de reorganizaciones societarias. No obstante, estas competencias deben estar integradas a las demandas del entorno económico. Por eso, una profesión que no evolucione con el entorno está destinada a desaparecer, debe reinventarse y adaptarse a nuevos retos y obligaciones del mercado, involucrando de manera activa a los contadores.

La información transversal; el profesional contable cuenta con acceso a información transversal de las compañías. Esta característica cumple con un factor diferenciador de distintas profesiones: buscar explotar el potencial que esto implica, lo cual se hace imperativo, pues a lo largo de este trabajo se evidencian 
herramientas extracontables que se encuentran dentro del campo de acción del contador o que por lo menos no son temas absolutamente ajenos a la profesión, lo cual le da una perspectiva siempre global de toda la operación. Hacer uso de esta información transversal en los esfuerzos de compañías colombianas del sector automotor para el acceso a estas CGSA, por parte del contable, es una propuesta que sin otro fin busca explotar las actividades que puedan influir activamente en las decisiones de negocio, participar en la planeación de los procesos productivos, revelar aspectos externos dentro de una CGS que influyan en los resultados financieros, tomar parte en la planeación de estudios de precios de transferencia, analizar y apoyar procesos jurídicos con valor probatorio, estructurar CS y CGS, y medir los efectos fiscales dentro de la operación. Los anteriores, son planteamientos que este estudio considera al alcance de las competencias de la profesión.

La contribución de la profesión contable en los objetivos de las CGS; es esencial para la profesión contable considerar igualmente su contribución, directa e indirectamente en el alcance de estos objetivos. Se necesita articular cómo la contabilidad y la profesión faciliten tal alcance y reconocer posibilidades de mejora. La IFAC (2017) sostiene que un paso en la dirección correcta es consolidar una profesión sólida, de principios éticos muy fuertes y definidos. Para la profesión es un gran paso mejorar en esta área, si se quiere llevar una mejor percepción ante la sociedad y, por ende, mejores desempeños profesionales. Una mejora en la transparencia de la información financiera que maneje un contable contribuye a alcanzar objetivos en todos los niveles y las estructuras. Se debe tener presente que no solo es la verdadera base sobre la que la profesión se construye, sino también permite potenciar los aspectos del perímetro de la profesión, que toman especial relevancia en estas CGSA.

\section{CONCLUSIONES}

El análisis y ejecución del presente trabajo, lleva a las siguientes conclusiones:

Tener en cuenta la evolución del concepto de CS y CGS permite observar de forma general la función que cumplen las CS en la generación de ventajas competitivas para las compañías. Así mismo, diagnosticar de forma detallada la CS de una compañía posibilita tener la carta de navegación para un gerente y para un contable debe ser el esquema de oportunidades de generación de eficiencias de toda clase; especialmente se abordan las eficiencias fiscales, en las que un contable puede llegar a tener participación.

Comprender la importancia del uso de estas CS y de pertenecer a las CGS, ha sido parte de los objetivos de esta investigación; no obstante, en un mundo cada vez más competitivo, el análisis de proyección de crecimiento dentro de estas CGS debe hacerse cuidadosamente y siempre observando factores externos.

En cuanto a las posiciones de los organismos internacionales, se generan impactos considerables en la normativa colombiana. La conceptualización, normalización y adopción en sus leyes es una constante y lo que es importante para efectos de análisis de CGS, es tener presente que cada jurisdicción de una manera $u$ otra al incorporar estas posiciones en su legislación adopta su propia interpretación de dichos conceptos y normas, lo cual hace único el análisis por cada jurisdicción.

Sobre los aspectos fiscales considerados en esta investigación, se puede indicar que están vinculados a las CGSA, previendo la administración eficiente de impuestos, pues el objetivo principal es articular los procesos de la operación de la CGSA, con los componentes fiscales, al utilizar estas "herramientas" propuestas, como eficiencias para generar valor agregado, maximizar los beneficios y crear ventajas competitivas, tanto en la calidad como en los costos bajos de los productos o servicios disponibles a la inserción de estas CGSA.

El resultado es la importante relación de aspectos extrafiscales de la CGSA, con el componente de planeación de la operación. En este sentido, se facilitan de forma significativa las relaciones y negociaciones que influyen en oportunidades de acceso a estas CGSA; es decir, la administración eficiente de impuestos es potencialmente favorable a la inserción de las compañías del sector automotor a las Cadenas Globales de Suministro.

Empoderar al contable en esta inserción y tener las competencias, desde el manejo constante de la información transversal de una compañía, lo cual supone para el contador público tener el poder de interactuar con estas situaciones de manera siempre favorable para las empresas, al reconocer que este es un enfoque gerencial, que soporta en ello la total capacidad en competencias y rol fundamental para la toma de decisiones que aporte al crecimiento y el desarrollo de la economía del país con visión global y esfuerzos conjuntos para visualizar oportunidades reales para Colombia. 


\section{AGRADECIMIENTOS}

Los autores del presente trabajo agradecen a la Pontificia Universidad Javeriana de Bogotá, por el apoyo para la realización del proyecto de investigación y asignación de recursos para los productos de investigación.

\section{REFERENCIAS}

Acevedo, J. y Díaz, I., Exploration and exploitation in Latin American firms: The Determinants of organizational ambidexterity and the country effect, https://doi.org/10.4067/S0718-27242019000400006, Journal of Technology Management \& Innovation, 14(4), 6-16, (2019)

Acero, M., Cadenas de suministros globales, hoy una realidad en Colombia, Portafolio, http://www.portafolio.co (2006)

Ali, A., Mahfouz, A. y Arisha, A., Analysing supply chain resilience: integrating the constructs in a concept mapping framework via a systematic literature review, Supply Chain Management: An International Journal, 22(1), 16-39 (2017)

Ballesteros, D., y Ballesteros, P., La logística competitiva y la administración de la cadena de suministros, DOI: 10.22517/23447214.7347, Scien-tia Et Technica, 10 (24), 201-206 (2004)

Barbosa, J. y Oundjan, A., La tributación en los negocios internacionales, Revista de derecho privado, 42, pp. 4-33 (2009)

Bermeo, L., Lizarazo, D., Villamil, N. y Vargas, K., Compraventa internacional de mercaderías, https://www.javeriana.edu.co (2005)

Carpio, R., La planificación tributaria internacional, RETOS, Revista de Ciencias de la Administración y Economía, 2(3), pp. 53-67 (2012)

Daniels, J., Radebaugh L., y Sullivan, D., Negocios Internacionales, Ambiente y Operaciones. 13ª Edición, 606-770, Pearson Prentice Hall, México D. F., México (2013)

De La Hoz, E.; Morelos, J. y López, L., Evaluación de la competitividad comercial del sector industrial colombiano mediante el coeficiente de apertura exportadora, Aglala, 10(1), 180-195 (2019)

DIAN: Dirección de Impuestos y Aduanas Nacionales (Colombia), Concepto 071190, 30 de septiembre, Bogotá-Colombia (2005)

DIAN: Dirección de Impuestos y Aduanas Nacionales (Colombia), Concepto 59714, 22 de octubre, Bogotá-Colombia (2014)

Drtina, R., y Correa, H. L., How transfer prices can affect a supply chain strategic decisión, DOI: 10.1504/IJLSM.2011.039595, International Journal of Logistics Systems and Management (IJLSM), Vol. 8, No. 4, (2011)

Gallardo, J., Admininstración Estratégica: de la Visión a la Ejecución, 1a Edición, 320-324, Alfa Omega, México D. F., México (2012)

Giraldo-García, J. A., Castrillón-Gómez, O. D., \& Ruiz-Herrera, S., Simulación discreta y por agentes de una cadena de suministro simple incluyendo un sistema de información geográfica (SIG), https://dx.doi.org/10.4067/S071807642019000600123, Información tecnológica, 30(6), 123-136, (2019)

Heredia Pérez, J., Geldes, C.,Kunc, M. \& Flores, A., New approach to the innovation process in emerging economies: The manufacturing sector case in Chile and Perú, https://doi.org/10.1016/j.technovation.2018.02.012, Technovation, (79), 3555, (2019)

Herrera, G., Análisis de modelos de planificación colaborativa en la cadena de suministros: Una revisión de la literatura, 12th Latin American and Caribbean Conference for Engineering and Technology (LACCEl'2014), "Excellence in Engineering to Enhance a Country's Productivity", Julio 22-24, Guayaquil, Ecuador (2014)

IFAC: International Federation of Accountants, Developing a future-ready profession, https://www.ifac.org (2017)

IMA: Institute of Management Accountants, Value chain analysis for assessing competitive advantage, https://www.imanet.org (1996)

Jüttner, U. y Maklan, S., Supply chain resilience in the global financial crisis: an empirical study, Supply Chain Management: An International Journal, 16(4), 246-259 (2011)

Kamalahmadi, M. y Parast, M.M., A review of the literature on the principles of enterprise and supply chain resilience: major findings and directions for future research, International Journal of Production Economics, 171, 116-133 (2016)

Monroy, M., El concepto clúster, ¿expectativas creadas o realidades posibles? El caso Medellín, Colombia, Cuadernos CLACSO-CONACYT \# 4, 4-7, Ciudad Autónoma de Buenos Aires, Argentina: CLACSO (2016)

Nogueira, M. G. S. y Hallal, D. R., Resiliência organizacional como capacidade estratégica para um melhor desempenho: um estudo em empresas de tecnologia da informação da cidade de Caçador/SC, 1-12. In VI Encontro de Estudos em Estratégia. Bento Gonçalves-Brasil (2013)

OCDE: Organización para la Cooperación y el Desarrollo Económicos, Global Value Chains: challenges, opportunities and implications for policy. Report prepared for submission to the G20 Trade Ministers Meeting, Sídney, Australia, 19 de julio (2014) 
OMC: Organización Mundial del Comercio, Acuerdo General sobre Aranceles Aduaneros y Comercio de 1994 (GATT, 1994), https://www.wto.org (1994)

Osorio, G., Cadena Global de suministro automotriz, Tercer (3er) Congreso Internacional en Supply Chain Management en una Economía Global: Interdisciplinariedad en las Ciencias Económicas, Contables y de Ingeniería, un Diálogo entre Sostenibilidad y Sustentabilidad, Bogotá, Colombia (2017)

Persson, G., Organization design strategies for business logistics, https://doi.org/10.1108/EUM0000000004332, International Journal of Physical Distribution \& Materials Management, Vol. 27 No. 5/6, pp. 282-291, (1997)

Porter, M. E., The competitive advantage: Creating and sustaining superior performance, Nueva York, Estados Unidos, Free Press (1998)

Presidencia de la República de Colombia, Decreto $N .{ }^{\circ} 2120$ por el cual se modifican los artículos 1.2.2.1.2. y 1.2.2.1.3. del Capítulo 1 del Título 2 de la Parte 2 del Libro 1; se sustituyen los Capítulos 2, 3 y 4 del Título 2 de la Parte 2 del Libro 1 y el Capítulo 1 del Título 3 de la Parte 6 del Libro 1 y se modifica el epígrafe del Capítulo 1 del Título 2 de la Parte 2 del Libro 1 del Decreto 1625 de 2016, Único Reglamentario en Materia Tributaria, Bogotá, Colombia, diciembre 15 (2017)

Presidencia de la Republica de Colombia, Ley $N .{ }^{\circ} 1943$, por el cual se expiden normas de financiamiento para el restablecimiento del equilibrio del presupuesto general y se dictan otras disposiciones, Bogotá, Colombia, diciembre 28 (2018)

Ramírez, M. C., Rodríguez, M de P., y González, J. P., Revisión de la literatura sobre el enfoque estratégico de los modelos de negocios, https://dx.doi.org/10.4067/S0718-07642019000600177, Información tecnológica, 30(6), 177-192, (2019)

Shunko, M., Do, H. T., \& Tsay, A. A., Supply chain strategies and international tax arbitraje, https://doi.org/10.1111/poms. 12629, Production and Operations Management, Vol. 26, No. 2, pp. 231-251, (2016)

Torre, V., Problemas de precios de transferencia de bienes intangibles en las empresas multinacionales, Boletín Mexicano de Derecho Comparado, №128, pp. 845-893 (2010)

WBG: World Bank Group, Measuring and analyzing the impact of GVCs on economic development, https://www.wto.org, Global Value Chain Development Report (2017)

Zamora, A. I., y González, J., Factores clave de la cadena logística del comercio exterior de un puerto mexicano: análisis a través de redes neuronales artificiales, http://dx.doi.org/10.22201/fca.24488410e.2018.1494, Contaduría y Administración, 64 (2), 1-19, (2018) 
